\title{
Fark Denklem Sistemleriyle Oluşturulmuş Ot-Otçul Modelinin Çatallanma Analizi
}

\author{
Şenol KARTAL
}

ÖZET: Bu çalışmada, fark denklem sistemiyle oluşturulmuş bir ot-otçul matematiksel modeli göz önüne alınmıştır. Center Manifold teoremi kullanılarak sistemde Neimark Sacker çatallanmasının oluşması için gerekli olan özdeğer eşliği, transversality ve nonresonance koşulları analiz edilmiş ve teorik olarak bu koşulların sağlandığı gösterilmiştir. Elde edilen bu teorik koşulların doğruluğunu göstermek için bazı parametre değerleri belirlenmiş ve bu parametre değerleri için sistemin çatallanma diagramı, faz diyagramları elde edilmiştir.

Anahtar Kelimeler: Fark denklem sistemi, kararlılık, Neimark-Sacker çatallanma, ot-otçul model

\section{Bifurcation Analysis of a Plant-Herbivore Model Constructed with System of Difference Equations}

\begin{abstract}
In this study, a plant-herbivore mathematical model constructed with the system of difference equation is considered. Using Center Manifold theorem, the eigenvalue assignment, transversality and nonresonance conditions that required for the existence of Neimark Sacker bifurcation in the system are analyzed and it has been theoretically shown that these conditions are satisfied. In order to show the accuracy of these theoretical conditions, some parameter values have been determined and the bifurcation diagram and phase diagrams of the system have been obtained for these parameter values.
\end{abstract}

Keywords: Neimark-Sacker bifurcation, plant-herbivore model, stability, system of difference equation

Şenol KARTAL (0000-0003-1205-069X), Nevşehir Hacı bektaş Veli Üniversitesi, Eğitim Fakültesi, Matematik ve Fen Bilimleri Eğitimi Bölümü, Nevşehir, Turkey

Sorumlu yazar/Corresponding Author: Şenol KARTAL, senol.kartal@ nevsehir.edu.tr 


\section{GíRiş}

Temelde ot (bitki)-otçul etkileşimine matematiksel olarak av-avcı modeli olarak yaklaşılmıştır (Caughley, 1981; May, 2001). Literatürde bu etkileşimi tanımlamak için birçok yazar kesikli ve sürekli dinamik sistemleri kullanmışlardır (Li, 2011; Chattopadhayay et al., 2001; Mukherjee et al., 2011; Danca et al., 1997; Agiza et al.,

$$
\left\{\begin{array}{l}
\frac{d x}{d t}=r x(t)\left(1-\frac{x(t)}{K}\right)-\alpha x(t) y(t) \\
\frac{d y}{d t}=-s y(t)+\beta x(t) y(t)
\end{array}\right.
$$

Burada $x(t)$ ve $y(t)$ sırasıyla ot ve otçul popü-lasyonunu temsil etmektedir. $r, K$ ve $\alpha$ parametreleri sırasıyla bitki büyüme oranı, bitki taşıma kapasitesi ve bitkinin otçul tarafından avlanma oranıdır. s otçulun ölüm oranı ve $\beta$ otçulun dönüşüm oranıdır (Chattopadhayay et al., 2001).

Deneysel veriler göstermiştir ki bitki ya da otçul non-overlapping nesillere sahip oldukları için bu etkileşim sürekli zamanlı dinamik sistemlerden ziyade kesikli zamanlı dinamik sistemlerle modellenmelidir. Dolayısıyla birçok yazar bu biyolojik gerçekten hareketle ot-otçul etkileşimi için fark denklem sistemlerini kullanmayı tercih etmişlerdir (Mukherjee et al., 2011; Danca et al., 1997; Agiza et al., 2009; Sui et

$$
\left\{\begin{array}{l}
\frac{d x}{d t}=r x(t)\left(1-\frac{x(t)}{K}\right)-\alpha x(t) y(\llbracket t \rrbracket), \\
\frac{d y}{d t}=-s y(t)+\beta x(\llbracket t \rrbracket) y(t),
\end{array}\right.
$$

(2) tam değer fonksiyonlu diferansiyel denklem sistemi hem sürekli zamanı hem de kesikli zamanı birlikte içerir ve gecikmeli diferansiyel denklemlerle yakından ilişkilidir. Çalışma (Kartal, 2016) de (2)
2009; Sui et al., 2007; Sun et al., 2014; Das and Sarkar, 2001; Cejas et al., 2004; Kartal, 2016). Li (Li, 2011) herbivore üzerindeki plant toksin etkisini incelemek için Holling type II fonksiyonu içeren diferansiyel denklem sistemi kullanmıştır. Chattopadhayay ve arkadaşları (Chattopadhayay et al., 2001) ise aşağıdaki sürekli zamanlı modeli önermişlerdir. al., 2007). Ot-Otçul modellemesine diğer bir yaklaşım gecikmeli diferansiyel denklemlerin kullanılmasıdır (Sun et al., 2014; Das and Sarkar, 2001; Cejas et al., 2004). Bu denklemlerin ot-otçul modellemesinde kullanılması otçulun bitki üzerinde oluşturduğu zarar ve bitkinin bu zarar karşılık gerçekleştirdiği savunma mekanizmasından kaynaklanmaktadır. Sun ve arkadaşları (Sun et al., 2014) gecikme zamanı içeren reaksiyon-difüzyon kısmi diferansiyel denklemlerini kullanarak ot-otçul etkileşimini incelemişlerdir.

Kartal (Kartal, 2016) ise (1) denklemine tam değer fonksiyonu katarak hem sürekli zamanlı hem de kesikli zamanlı aşă̆ıdaki modeli oluşturmuş̧tur. denklem sisteminin $t \in[n, n+1)$ alt aralıklarında çözümünden aşă̆ıdaki fark denklem sistemi elde edilmiştir.

$$
\left\{\begin{array}{l}
x(n+1)=\frac{x(n)[r-\alpha y(n)]}{[r-\alpha y(n)-r k x(n)] e^{-[r-\alpha y(n)]}+r k x(n)} \\
y(n+1)=y(n) e^{\beta x(n)-s}
\end{array}\right.
$$


$\mathrm{Bu}$ çalışmada ise Center Manifold teorisi kullanılarak (3) sisteminin Neimark-Sacker çatallanma analizi yapılacaktır.

\section{MATERYAL VE YÖNTEM}

(3) sisteminin çatallanma analizine geçmeden önce yerel kararlılık analizi sonuçlarını bilmek gereklidir. (3) sisteminin pozitif denge noktas 1

$\beta>k S$

koşulu altında

$E^{*}=\left(x^{*}, y^{*}\right)=\left(\frac{s}{\beta}, \frac{r}{\alpha}\left(1-\frac{k s}{\beta}\right)\right)$

olarak elde edilebilir. $E^{*}$ denge noktasında (3) sisteminin $J^{*}$ jakobyen matrisi ise

$J^{*}=\left(\begin{array}{cc}e^{-\frac{k r s}{\beta}} & \frac{\left(-1+e^{-\frac{k r s}{\beta}}\right) \alpha}{k r} \\ -\frac{k r s-r \beta}{\alpha} & 1\end{array}\right)=\left(\begin{array}{cc}e^{-k r x^{*}} & \frac{\left(-1+e^{-k r x^{*}}\right) \alpha}{k r} \\ \beta y^{*} & 1\end{array}\right)$

olarak hesaplanır. (6) Jakobyen matrisinin karakteristik denklemi ise

$p(\lambda)=\lambda^{2}+\lambda\left(-1-e^{-k r x^{*}}\right)+e^{-k r x^{*}}+\frac{\left(1-e^{-k r x^{*}}\right) \alpha \beta y^{*}}{k r}=0$

olarak bulunur (Kartal, 2016). (7) karakteristik denkleminden elde edilecek olan $\lambda_{\mathrm{i}}, \mathrm{i}=1,2$ özdeğerlerinin 1 den küçük olmasını sağlayan koşullar ya da diğer bir deyişle (3) matematiksel modelinin $E^{*}$ denge noktasının yerel asimptotik kararlı olmasını sağlayan koşullar

$$
k s<\beta<k+k s \quad \text { ise. }
$$

\section{BULGULAR VE TARTIŞMA}

$\mathrm{Bu}$ bölümde (3) sisteminin Neimark-Sacker çatallanma analizi yapılacaktır. Neimark-Sacker çatallanması, kesikli zamanlı dinamik modellerde ortaya çıkan bir çatallanmadır. Bu çatallanma tipi için linerleştirilmiş sistemin jakobyen matrisinin karakteristik denklemi birim dairenin üzerinde olan bir çift eşlenik kompleks özdeğere sahiptir ve karakteristik denklemin diğer bütün özdeğerleri birim dairenin içindedir. (3) fark denklem sisteminin Neimark-Sacker
Schur-Cohn kriterlerinin kullanılmasıyla aşağıdaki gibi elde edilmiştir.

Teorem 1 (Kartal, 2016): (3) matematiksel modelinin denge noktası yerel asimptotik kararlıdır ancak ve ancak

çatallanma analizini yapabilmek için Kuznetsov'un (Kuznetsov, 1998) ortaya koyduğu ve bir çok yazarın kullandığ çatallanma teorisi kullanılacaktır (Kuznetsov, 1998; Xin et al., 2009; Wen, 2005; Peng, 2005; He and Li, 2014; Sohel Rana, 2015). Aşağıdaki teorem iki boyutlu kesikli zamanlı dinamik sistemler için Neimark-Sacker Çatallanma kriterlerini vermektedir.

Teorem 2 (Xin et al., 2009): (İki boyutlu kesikli zamanlı dinamik sistemler için Neimark-Sacker Çatallanma Kriterleri) 
İki boyutlu

$x_{k+1}=f\left(x_{k}, \mu\right)$

kesikli dinamik sistemini göz önüne alalım. Burada $x_{k+1}, x_{k} \in R^{2}$ vektörler ve $\mu$ de bir parametredir.

(A1) (8) sisteminin $\mathrm{x}^{*}$ denge noktasındaki $J_{x_{k}} f\left(x^{*}, \mu\right)$ jakobyen matrisinin karakteristik denklemi $p(\lambda)=\lambda^{2}+p_{1} \lambda+p_{0}=0$,

(A2) (8) sisteminin normalleştirilmiş denklemleri

$\tilde{z}=e^{i \theta_{0}} z+\frac{1}{2} g_{20} z^{2}+g_{11} z \bar{z}+\frac{1}{2} g_{02} \bar{z}^{2}+\frac{1}{2} g_{21} z^{2} \bar{z}$,

(A3) Birinci Lyapunov katsayısı

$a(0)=\operatorname{Re}\left[\frac{e^{-i \theta_{0}} g_{21}}{2}\right]-\operatorname{Re}\left[\frac{\left(1-2 e^{i \theta_{0}}\right) e^{-2 i \theta_{0}}}{2\left(1-e^{i \theta} 0\right)} g_{20} g_{11}\right]-\frac{1}{2}\left|g_{11}\right|^{2}-\frac{1}{4}\left|g_{02}\right|^{2}$

olmak üzere

(B1) Özdeğer eşliği : $\mu=\mu^{*}$ noktasında $1+p_{1}+p_{0}>0,1-p_{1}+p_{0}>0,1+p_{0}>$ $0,1-p_{0}=0$,

(B2) Transversality Koşulu: $\left.\frac{d\left|\lambda_{i}(\mu)\right|}{d \mu}\right|_{\mu=\mu^{*}} \neq 0$,

(B3) Bir çift eşlenik kompleks özdeğerler $\lambda_{1}$ ve $\lambda_{2}$ için resonance koşulu $\lambda_{1}{ }^{m}\left(\mu^{*}\right)=1$ ya da nonresonance koşulu $\lambda_{1}{ }^{m}\left(\mu^{*}\right) \neq 1, m=3,4,5, \ldots \ldots$

koşulları sağlansın.

$\mathrm{Bu}$ durumda $\mu=\mu^{*}$ noktasında Neimark-Sacker çatallanması oluşur. Nonresonance koşulu altında eğer $a(0)<0$ ise supercritical Neimark-Sacker çatallanması, diğer durumda subcritical Neimark-Sacker çatallanması oluşur.

Teorem 3: Eğer

$k s<\beta^{*}=k+k s$ 
ise bu durumda (3) sistemi için denge noktasında Neimark-Sacker çatallanması oluşur. Üstelik $\alpha(0)$ $<0$ ise bu durumda oluşan çatallanma supercritical Neimark-Sacker çatallanması, diğer durumda ise subcritical Neimark-Sacker çatallanmasıdır.

İspat. Öncelikle (B1) özdeğer eşliğininin koşullarını göz önüne alalım. (7) karakteristik denkleminden

$p_{1}=-1-e^{-k r x^{*}}$ ve $p_{0}=e^{-k r x^{*}}+\frac{\left(1-e^{-k r x^{*}}\right) \alpha \beta \bar{y}}{k r}$

dir.

$1+p_{1}+p_{0}>0,1-p_{1}+p_{0}>0$ ve $1+p_{0}>0$ dan sirasiyla

$\frac{\left(1-e^{-k r x^{*}}\right) \alpha \beta \bar{y}}{k r}>0$,

$2+2 e^{-k r x^{*}}+\frac{\left(1-e^{-k r x^{*}}\right) \alpha \beta y^{*}}{k r}>0$

ve

$1+e^{-k r x^{*}}+\frac{\left(1-e^{-k r x^{*}}\right) \alpha \beta y^{*}}{k r}>0$

elde edilir ki bu eşitsizlikler (4) eşitsizliğinin varlığı altında her zaman mevcuttur. $1-p_{0}=0$ denklemi $\beta$ ya gore çözülürse $\beta^{*}=k+k s$ elde edilir.

Öte yandan (6) matrisinin özdeğerleri

$\lambda_{1,2}(\beta)$

$=\frac{e^{-k r x^{*}}\left(k+e^{k r x^{*}} k \mp \tilde{u} \sqrt{\left(-1+\mathbb{e}^{k r x^{*}}\right) k\left(k\left(1-\mathbb{e}^{k r x^{*}}(1+4 s)\right)+4 \mathbb{e}^{k r x^{*}} \beta\right)}\right)}{2 k}$

şeklinde hesaplanır ve $\beta=\beta^{*}$ için bu kompleks özdeğerler

$\lambda_{1,2}\left(\beta^{*}\right)=\frac{1}{2} e^{-\frac{r s}{1+s}}\left(1+e^{\frac{r s}{1+s}} \mp \underline{n} \sqrt{-1-2 e^{\frac{r s}{1+s}}+3 e^{\frac{2 r s}{1+s}}}\right)$

olur. Buradan kolayca görülebilir ki $\left|\lambda_{1,2}\left(\beta^{*}\right)\right|=1$ dir. Diğer taraftan (B2) koşulundan 
$\lambda_{1,2}(\beta)$

$=\frac{e^{-k r x^{*}}\left(k+e^{k r x^{*}} k \mp \tilde{u} \sqrt{\left(-1+e^{k r x^{*}}\right) k\left(k\left(1-\mathbb{e}^{k r x^{*}}(1+4 s)\right)+4 \mathbb{e}^{k r x^{*} \beta}\right)}\right)}{2 k}$

şeklinde hesaplanır ve $\beta=\beta^{*}$ için bu kompleks özdeğerler

$\lambda_{1,2}\left(\beta^{*}\right)=\frac{1}{2} e^{-\frac{r s}{1+s}}\left(1+e^{\frac{r s}{1+s}} \mp \sqrt[g]{\left.-1-2 e^{\frac{r s}{1+s}}+3 e^{\frac{2 r s}{1+s}}\right)}\right.$

olur. Buradan kolayca görülebilir ki $\left|\lambda_{1,2}\left(\beta^{*}\right)\right|=1$ dir. Diğer taraftan (B2) koşulundan

$\left.\frac{d\left|\lambda_{i}(\beta)\right|}{d \beta}\right|_{\beta=\beta^{*}}=\frac{e^{-2 k r x^{*}}\left(-k s+e^{k r x^{*}} s(k s-\beta)\right)}{\sqrt{2} \beta \sqrt{1+e^{-2 k r x^{*}}+2 s-\frac{2 \beta}{k}+e^{-k r x^{*}}\left(-2 s+\frac{2 \beta}{k}\right)}} \neq 0$

elde edilir ki bu bize Transversality koşulunun sağlandığını gösterir. Diğer taraftan $\operatorname{trJ}\left(r_{2}\right)=-p_{1} \neq 0,-1$, nonresonance koşulundan

$1+e^{-k r x^{*}} \neq 0,-1$,

elde edilir ki bu da

$\lambda^{k}{ }_{i}\left(\beta^{*}\right) \neq 1, \quad k=1,2,3,4$.

olması demektir. Sonuç olarak (3) sistemi için $k s<\beta^{*}=k+k s$ koşulu altında Neimark-sacker çatallanması mevcuttur.

Şimdi ise çatallanmanının türünü belirlemek için $a(0)$ değerini hesaplayalım.

$q \in R^{2}, J\left(\beta^{*}\right) q=e^{i \theta_{0}} q$ olacak şekilde $J\left(\beta^{*}\right)$ Jakobyen matrisinin $\lambda_{1}\left(\beta^{*}\right)$ özdeğerine karşılık gelen özvektörü ve $p \in R^{2}$ de $J^{T}\left(\beta^{*}\right) p=e^{-i \theta_{0}} p$ olacak şekilde $J^{T}\left(\beta^{*}\right)$ matrisinin $\overline{\lambda_{1}\left(\beta^{*}\right)}$ özdeğerine karş̧lık gelen özvektörü olsun. Bu durumda

$q \sim\left(1, \frac{-\left(-1+e^{\frac{r s}{1+s}}\right) k r-\frac{\sqrt[b]{I}}{-1-2 e^{\frac{r s}{1+s}}+3 e^{\frac{2 r s}{1+s}} k r}}{2\left(-1+e^{\frac{r s}{1+s}}\right) \alpha}\right)^{T}$ 
ve

$p \sim\left(\frac{\left(-1+e^{\frac{r s}{1+s}}\right) k r+\sqrt[n]{-1-2 e^{\frac{r s}{1+s}}+3 e^{\frac{2 r s}{1+s}} k r}}{2\left(-1+e^{\frac{r s}{1+s}}\right) \alpha}, 1\right)^{T}$

dir. Buradan $\langle p, q\rangle=1$ olmasını sağlayan normalleştirilmiş vektörler

$q=\left(1, \frac{-\left(-1+e^{\frac{r s}{1+s}}\right) k r-\sqrt[b]{-1-2 e^{\frac{r s}{1+s}}+3 e^{\frac{2 r s}{1+s}} k r}}{2\left(-1+e^{\frac{r s}{1+s}}\right) \alpha}\right)^{T}$

ve

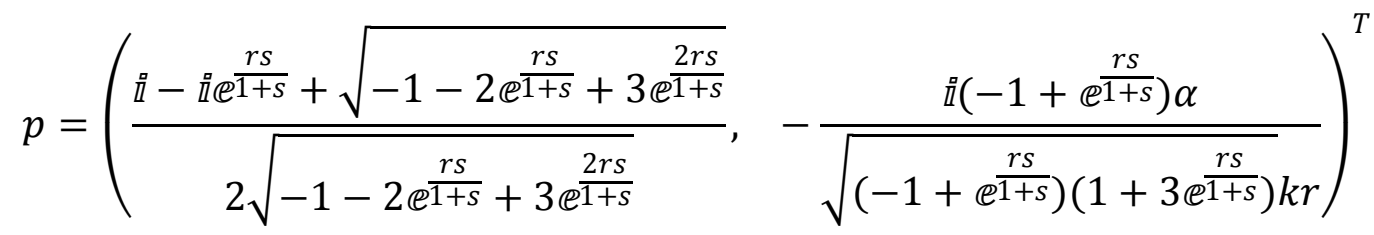

olarak seçilebilir.

$\left\{\begin{array}{l}x=x^{*}+x_{1} \\ y=y^{*}+x_{2}\end{array}\right.$

dönüşümü ile $E^{*}=\left(x^{*}, y^{*}\right)=\left(\frac{s}{\beta}, \frac{r}{\alpha}\left(1-\frac{k s}{\beta}\right)\right)$ denge noktası orjine taşınılabilir. Bu durumda (3) sistemi

$$
\left\{\begin{array}{l}
x_{1}(n+1)=\frac{e^{k r x^{*}}\left(s+\beta x_{1}(n)\right)\left(k r s-\alpha \beta x_{2}(n)\right)}{\beta\left(e^{k r x^{*}} k r\left(s+\beta x_{1}(n)\right)-e^{\alpha x_{2}(n)} \beta\left(k r x_{1}(n)+\alpha x_{2}(n)\right)\right)} \\
x_{2}(n+1)=\frac{e^{\beta x_{1}(n)}\left(r(-k s+\beta)+\alpha \beta x_{2}(n)\right)}{\alpha \beta},
\end{array}\right.
$$

halini alır. Şimdi

$x=z q+\bar{z} \bar{q}$

olacak şekilde 
$H(z, \bar{z})=<p, F\left(z q+\bar{z} \bar{q}, \beta^{*}\right)>$

fonksiyonunu oluşturalım. Bu fonksiyonun $(z, \bar{z})=(0,0)$ noktasındaki Taylor serisi

$$
H(z, \bar{z})=\sum_{2 \leq j+k \leq 3} \frac{1}{j ! k !} g_{j k} z^{j} \bar{z}^{-k}+O\left(|z|^{4}\right)
$$

şeklindedir ve $g_{j k}$ taylor katsayıları hesaplandıktan sonra çatallanmanın yönü

$$
a(0)=\operatorname{Re}\left[\frac{e^{-i \theta_{0}} g_{21}}{2}\right]-\operatorname{Re}\left[\frac{\left(1-2 e^{i \theta_{0}}\right) e^{-2 i \theta_{0}}}{2\left(1-e^{i \theta_{0}}\right)} g_{20} g_{11}\right]-\frac{1}{2}\left|g_{11}\right|^{2}-\frac{1}{4}\left|g_{02}\right|^{2}
$$

formülünden belirlenir.

Şimdi yukarıda elde edilen teorik sonuçları doğruluğunu nümerik olarak göstermeye çalışalım.

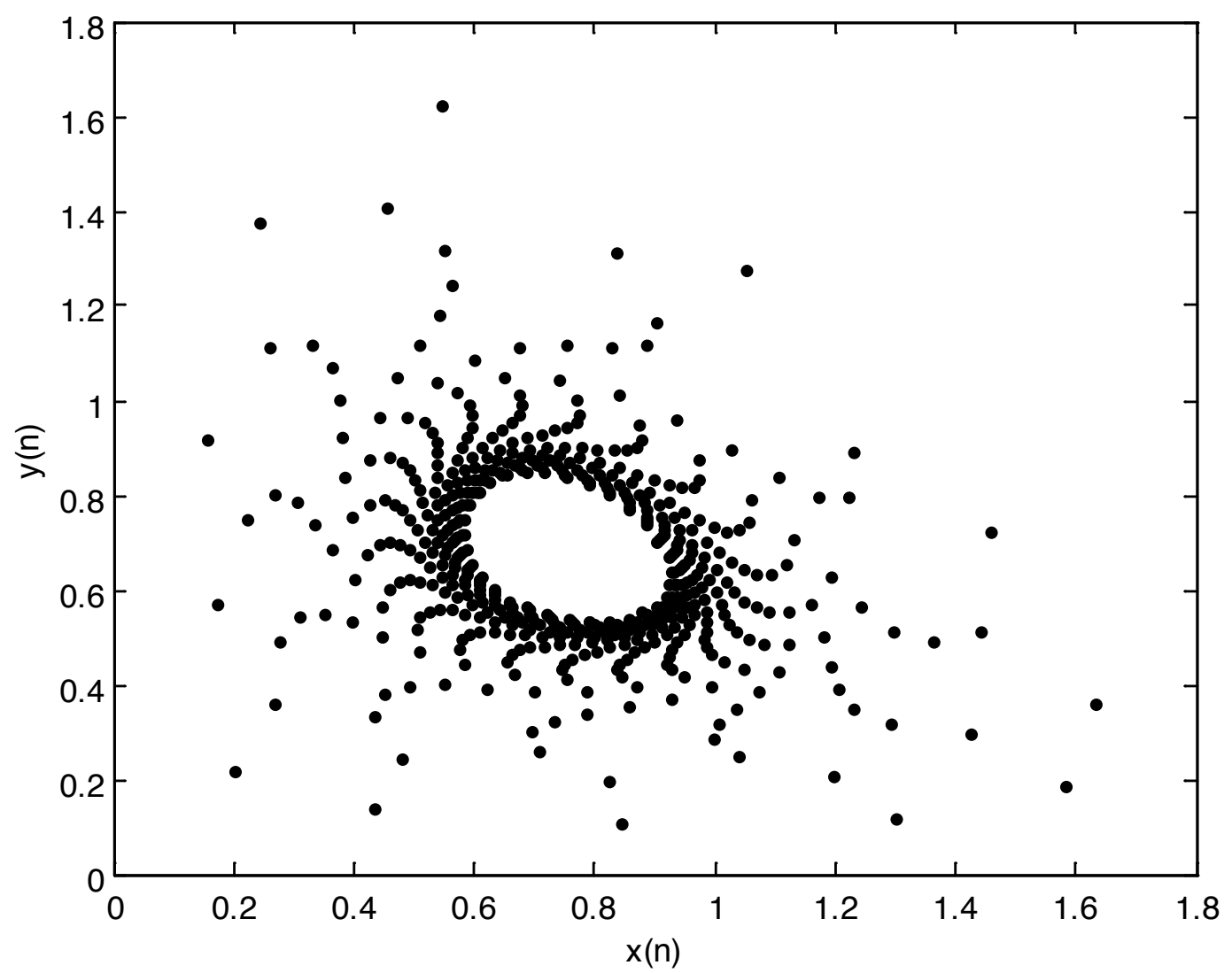

Şekil 1. $\beta^{*}=0.8$ değeri için sistemin Neimark-Sacker çatallanması. 
Örnek 1. $r=1.2, s=0.6, \alpha=1.1, k=0.5, \beta^{*}=0.8$ parametre değerleri için (20) sisteminin Jakobyen matrisi

$J\left(\beta^{*}\right)=\left(\begin{array}{cc}0.637628 & -0.664348 \\ 0.545454 & 1\end{array}\right)$

olarak hesaplanır ve bu matrisin özdeğerleri

$\lambda_{1,2}=0.818814 \mp 0.574059 i=e^{ \pm i \theta_{0}}, \quad \theta_{0}=0.611454$

şeklindedir. Burada $\left|\lambda_{1,2}\right|=1$ olduğu açıktır. Öte yandan $J\left(\beta^{*}\right)$ matrisinin özvektörleri (16) ve (17) eşitliklerinden

$q \sim(1, \quad-0.272727-0.864093 i)^{T}$

$p \sim(0.272727+0.864093 i, \quad 1)^{T}$

şeklinde belirlenebilir ve $\langle p, q\rangle=1$ olmasını sağlayan normalleştirilmiş vektörler ise (18) ve (19) dan

$q=(1, \quad-0.272727-0.864093 i)^{T}$

$p=(0.5-0.157811 i, \quad-0.57864 i)^{T}$

olarak hesaplanabilir. (21) ve (22) den ise $\mathrm{H}(\mathrm{z}, \overline{\mathrm{z}})$ fonksiyonunun $\mathrm{g}_{\mathrm{jk}}$ taylor katsayıları

$g_{20}=0.286335+0.374542 i, \quad g_{11}=0.0142984+0.00451291 i$

$g_{21}=0.647223-0.171596 i, \quad g_{02}=-1.00559-0.601554 i$

olarak bulunur. (23) eşitliğinden ise $a(0)=-0.132963$ olarak hesaplanır ki buda bize $\beta^{*}=0.8$ noktasında supercritical Neimark-Sacker çatallanmasının oluştuğunu gösterir. (Şekil 1, Şekil 2, Şekil 3) 


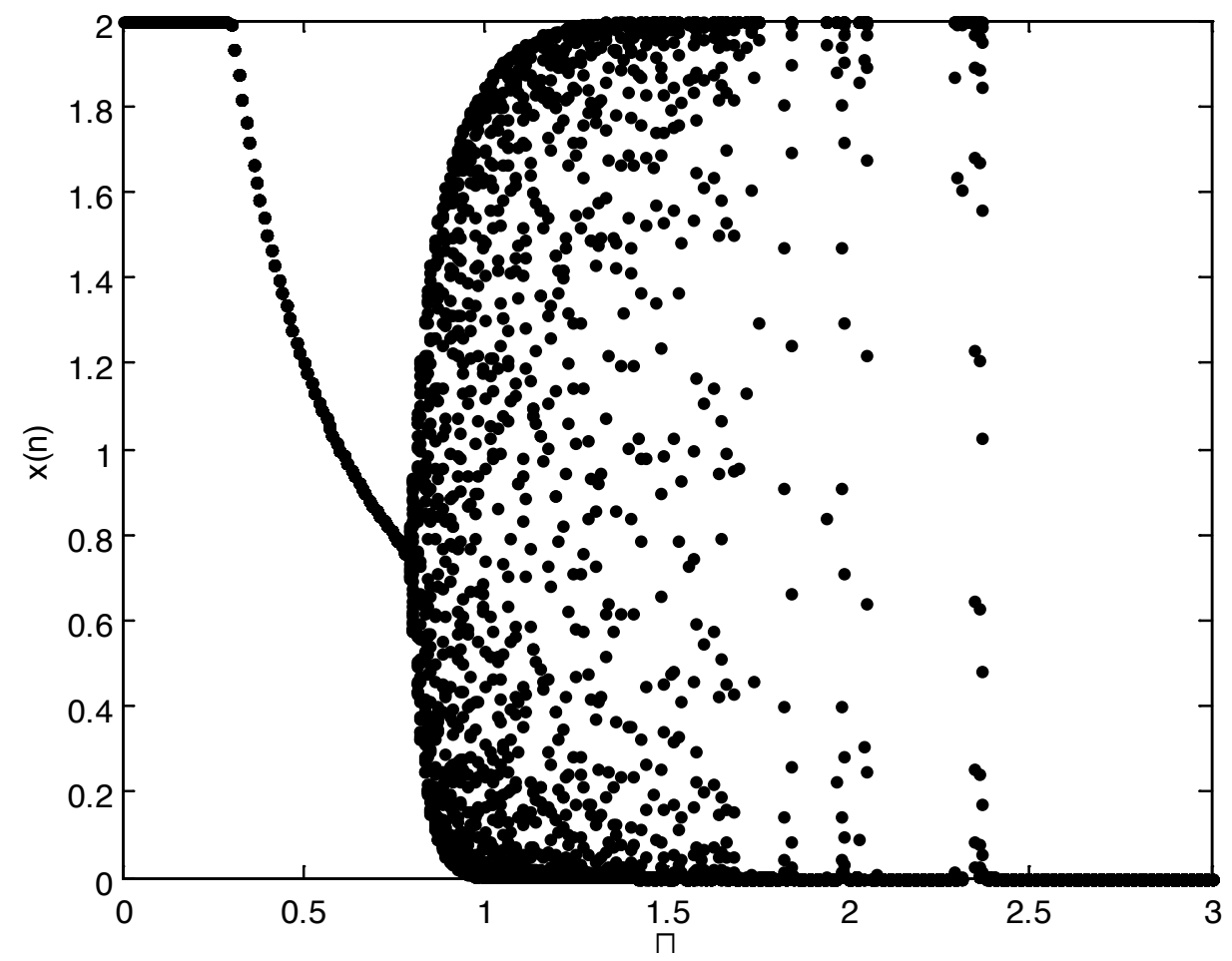

Şekil $2 \beta \in[0,3]$ ve $x(1)=2, y(1)=2$ başlangıç koşulları için $\beta$ ya karşı çizilmiş (3) sisteminin $x(n)$ popülasyonunun çatallanması.

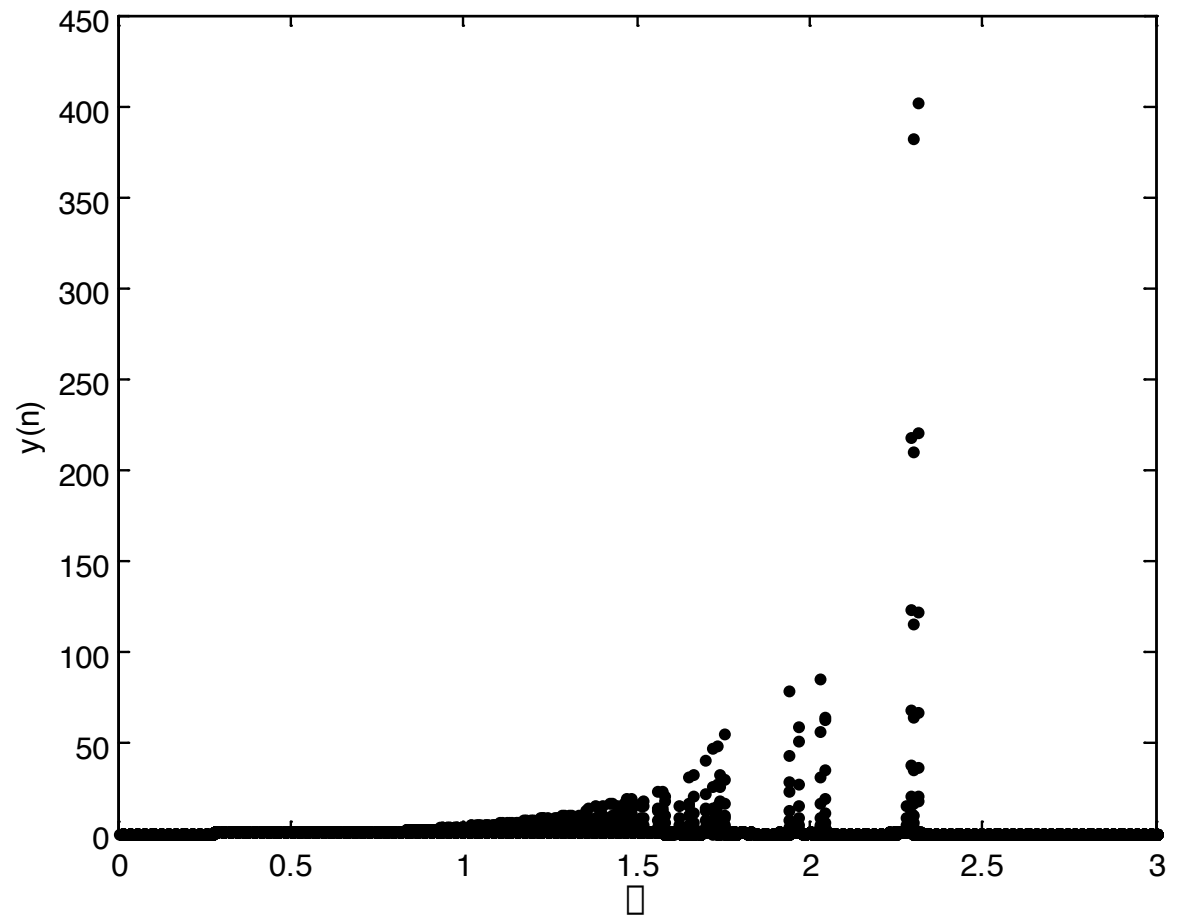

Şekil $3 \beta \in[0,3]$ ve $x(1)=2, y(1)=2$ başlangıç koşulları için $\beta$ ya karşı çizilmiş (3) sisteminin $y(n)$ popülasyonunun çatallanması. 


\section{SONUÇ}

Buçalışmada (2) tam değer fonksiyonlu diferansiyel denklem sisteminin $t \in[n, n+1)$ alt aralıklarında çözümünden (3) fark denklem sistemi elde edilmiştir. (3) fark denklem sisteminin pozitif denge noktasının

\section{KAYNAKLAR}

Agiza HN, Elabbasy EM, Metwally HE., Elsadany AA, 2009. Chaotic dynamics of a discrete prey-predator model with Holling type II. Nonlinear Anal Real, 10: 116-119.

Cejas VO, Fort J, Mendez V, 2004. The role of the delay time in the modeling of biological range expansions. Ecology, 85: 258264.

Caughley G, Lawton JH, 1981. Plant-herbivore systems, in Theoretical Ecolog. Sinauer Associates, Sunderland, 132-166.

Chattopadhayay J, Sarkar R, Hoballah MEF, Turlings TCJ, Bersier LF, 2001. Parasitoids may determine plant fitness-a mathematical model based on experimental data. J Theor Biol, 212: 295-302.

Danca M, Codreanu S, Bako B, 1997. Detailed analysis of a nonlinear prey-predator model. J Biol Phys, 23: 11-20.

Das K, Sarkar AK, 2001. Stability and oscillation of an autotrophherbivore model with time delay. Int J Sys Sci, 32: 585-590.

He Z, Li B, 2014. Complex dynamic behavior of a discrete time predator-prey system of Holling-III type. Adv Differ Equ, 180.

Kartal S, 2016. Dynamics of a plant-herbivore model with differential-difference equations. Cogent Mathematics, 3: Article Number: 1136198.

Kuznetsov YA, 1998. Elements of applied bifurcation theory. Springer-Verlag, Newyork. yerel asimptotik kararlı olmasını sağlayan koşullar Schur-Cohn kriterleri kullanılarak belirlenmiştir. Yine sistemde koşulu altında supercritical Neimark-Sacker çatallanmasının oluştuğu hem teorik hem de nümerik olarak gösterilmiştir.

May RM, 2001. Stability and complexity in model ecosystems. Princeton University Press, 40, New Jercy.

Li Y, 2011. Toxicity impact on a plant-herbivore model with disease in herbivores. Comput Math Appl, 62: 2671-2680.

Mukherjee D, Das P, Kesh D, 2011. Dynamics of a plant-herbivore model with holling type II functional response. Computational and Mathematical Biology, 2: 1-9.

Peng M, 2005. Multiple bifurcations and periodic "bubbling" in a delay population model. Chaos Soliton Fract, 25: 1123-1130.

Sui G, Fan M, Loladze I, Kuang Y, 2007. The Dynamics of a stoichiometric plant-herbivore model and its discrete analog. Math Biosci Eng, 4: 29-46.

Sun GQ, Chakraborty A, Liu QX, Jin Z, Anderson K.E., 2014. Influence of time delay and nonlinear diffusion on herbivore outbreak. Commun Nonlinear Sci Numer Simulat, 19: 15071518.

Sohel Rana SM, 2015. Bifurcation and complex dynamics of a discrete-time predator-prey system. Comput Ecol Softw, 5: 187-200.

Xin B, Ma J, Gao Q, 2009. The complexity of an investment competition dynamical model with imperfect information in a security market. Chaos Soliton Fract, 42: 2425-2438.

Wen GL, 2005. Criterion to identify Hopf bifurcations in maps of arbitrary dimension. Phys Rev E ,72: 026201-3. 